\title{
Retraction notice for: "Schizandrin A exerts anti-tumor effects on A375 cells by down-regulating H19" [Braz J Med Biol Res (2019) 52(10): e8385]
}

\author{
Yiming $\mathrm{Bi}^{1 \oplus}$, Yan $\mathrm{Fu}^{2 \oplus \bowtie}$, Shuyan Wang ${ }^{1 \oplus}$, Xingxiu Chen ${ }^{1 \oplus}$, and Xiaoping Cai ${ }^{1 \oplus}$ \\ ${ }^{1}$ Department of Oncology, Binzhou People's Hospital, Binzhou, China \\ ${ }^{2}$ Department of Dermatology, Binzhou People's Hospital, Binzhou, China
}

Retraction for: Braz J Med Biol Res | doi: 10.1590/1414-431X20198385 | PMID: 31618367 | PMCID: PMC6787960

The authors would like to retract the article "Schizandrin A exerts anti-tumor effects on A375 cells by down-regulating H19" that was published in volume 52, no. 10 (2019) (Epub Oct 10, 2019) of the Brazilian Journal of Medical and Biological Research.

After the publication of this study, the corresponding author requested its retraction due to "the identification of unspecified data inconsistency that could lead to mistaken conclusions." The Editors agreed with and endorsed that decision.

The Brazilian Journal of Medical and Biological Research had received authorization from all authors before the publication of the paper. We regret the unprofessional behavior of the authors involved. 


\title{
Schizandrin A exerts anti-tumor effects on A375 cells by down-regulating 419
}

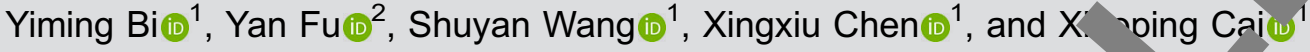 \\ ${ }^{1}$ Department of Oncology, Binzhou People's ' uptal, Bir. China \\ ${ }^{2}$ Department of Dermatology, Binzhou People's lospital, "'nzhou, China
}

\begin{abstract}
Malignant melanoma (MM) is one of the malignant tumors with highly metastatic and ags, essi hiological actions. Schizandrin $\mathrm{A}(\mathrm{SchA})$ is a bioactive lignin compound with strong anti-oxidant and anti-aging prope" $\mathrm{s}$, which stable at room temperature and is often stored in a cool dry place. Hence, we investigated the effects of Sc , or MM cell line A375 and its underlying mechanism. A375 cells were used to construct an in vitro MM cell model. Cell bilit " ation, apoptosis, and migration were detected by Cell Counting Kit-8, BrdU assay, flow cytometry, and transwell -chamber assay, respectively. The cell cycle-related protein cyclin D1 and cell apoptotic proteins (Bcl-2, Bax maved-ca. se-3, and cleaved-caspase-9) were analyzed by western blot. Alteration of H19 expression was achieved by non with pEX-H19. PI3K/AKT pathway was measured by detecting phosphorylation of PI3K and AKT. SchA significa $v$ ecreased cell viability in a dose-dependent manner. Furthermore, SchA inhibited cell proliferation and cyclin D1 expressi SchA increased cell apoptosis along with the up-regulation of pro-apoptotic proteins (cleaved-caspase-3, cleand-caspase , and Bax) and the down-regulation of antiapoptotic protein (Bcl-2). Besides, SchA decreased migratio ana wn-regulated matrix metalloproteinases (MMP)-2 and MMP-9. SchA down-regulated IncRNA H19. Overexpressic f H19 b ckaded the inhibitory effects of SchA on A375 cells. SchA decreased the phosphorylation of PI3K and AKT y ile ove kpression promoted the phosphorylation of PI3K and AKT. SchA inhibited A375 cell growth, migration, and PI?K/An athway through down-regulating H19.
\end{abstract}

Key words: Malignant melanoma; Schizandrin A;

\section{Introduction}

Malignant melanoma (MM) is or or the malignant tumors with highly metastatic and gressiv biological actions. Besides, MM accounted fo $\mathrm{s} 0-80$ of skincancer related deaths (1). Rerant studitu uve indicated that MM is not a single entity neoplasms with different causes ir gical behaviors, and outcomes (2). Unfortur des, e the development of technology and med ne, e sur val rate for advanced MM has not improved (3). Therefore, w me nes or therapies are urgently needed to tre In recunt years, more attention has been given to traditi 1 Chinese medicine for novel potential treatr int.

$\mathrm{Sc}^{\prime}$ and $\mathrm{A}^{\prime} \mathrm{A}(\mathrm{Sch} \mathrm{A})$, extracted from Fructus schisandra, has n pr en to play effective functions in various di $s \mathrm{du}$ its biological properties (4). For example, chA alloviated lipopolysaccharide-induced inflammaajury in human keratinocyte HaCat cells (5). SchA inci ced chemosensitivity to 5 -fluorouracil by upregulation or miR-195 in colon cancer cells (6). Importantly, previous studies have reported that SchA possesses anti-tumor activities (7). Hence, we hypothesized that SchA might play a vital role in MM.

In recent years, long non-coding RNAs (IncRNAs) have received considerable attention due to their important functions in regulating gene expression in multiple approaches, such as chromosome remodeling, transcription, and post-transcriptional processing (8). Increasing evidence has proven that IncRNAs are closely associated with tumor development or progression (9). For example, IncRNA plasmacytoma variant translocation 1 (PVT1) participates in prostate cancer cell development and growth (10). LncRNA maternally expressed gene (MEG) 3 alleviates gastric cancer cell proliferation and metastasis (10). Among all the identified IncRNAs, IncRNA H19 was characterized as an oncogenic gene in diverse cancers, and the potentiated expression of $\mathrm{H} 19$ is closely related to tumor genesis and development (11). Importantly, IncRNA H19 was reported to promote glucose metabolism and cell growth in MM through regulating miR-106a-5p/E2F3 axis (12). Furthermore, the elevated expression of IncRNA H19 predicted poor outcomes for $\mathrm{MM}$ via regulating cell growth, invasion,

Correspondence: Yan Fu: <fuyan0059@sina.com>

Received January 3, 2019 | Accepted August 14, 2019 
and migration (13). Therefore, we inferred that SchA might play its roles in MM cell lines through regulating IncRNA H19.

In our study, we used A375 cells to construct a MM model in vitro and investigated the effects of SchA on A375 cells and its underlying mechanisms.

\section{Material and Methods}

\section{Cell culture and treatment}

The MM cell line A375 (ATCC ${ }^{\circledR}$ CRL-1619 ${ }^{\mathrm{TM}}$ ) was purchased from American Type Culture Collection (ATCC, USA). The culture medium for A375 cells was Dulbecco's modified Eagle's medium (DMEM, ATCC, Cat. No. 30-2002) supplemented with $10 \%$ fetal bovine serum (FBS, Gibco, USA). The cells were maintained in the environment with $5 \% \mathrm{CO}_{2}$ and $37^{\circ} \mathrm{C}$.

SchA ( $\geqslant 98.0 \%$ (HPLC), Figure 1) was obtained from Sigma-Aldrich (USA). SchA was diluted in dimethylsulfoxide (DMSO) to $0-50 \mu \mathrm{M}$. The cells were treated with SchA for $24 \mathrm{~h}$.

\section{Cell viability assay}

Cell Counting Kit-8 (CCK-8, Yeasen, China) was used for examining cell viability. Treated A375 cells were seeded in a 96-well plate at the density of $2 \times 10^{5}$ cells/well, 1 der proper conditions $\left(37^{\circ} \mathrm{C}\right.$ and $\left.5 \% \mathrm{CO}_{2}\right)$. Then, $10 \mu \mathrm{l} \mathrm{CK}$. solution was added and cells were incubated for $1 \mathrm{~h}$. or incubation, absorption was read at 450 Microplate Reader (Bio-Rad, USA).

\section{Proliferation assay}

Bromodeoxyuridine (BrdU, Sigm Aldrich was used for cell proliferation assay. In brief, $A 3$ cells ated with SchA or co-treated with SchA and trans with $\mathrm{pEX}$ $\mathrm{H} 19$ were plated in a 96-well $\mathrm{p}$. C. $\mathrm{BrdU}(1 \mathrm{mg} / \mathrm{mL})$ was added to the cultured cells. Nere then incubated for $3 \mathrm{~h}$ and proliferated 's we labeled. Finally, cells

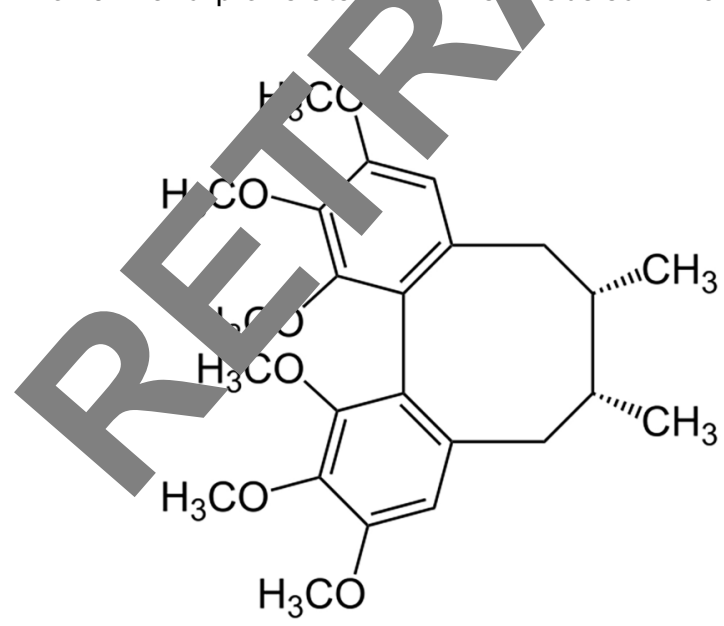

Figure 1. Molecular formula of schizandrin A. incorporated with BrdU were quantified using a BrdU cell proliferation assay kit (Roche Diagnostics, USA)

\section{Cell apoptosis assay}

Propidium iodide (PI) and fluorescein isothi an (FITC)-conjugated annexin $\mathrm{V}$ staining (Yeasen, $Q$,a) were used for cell apoptosis assay. In of, cell at the density of 100,000 cells/well were ed in plate. Treated cells were washed twice ih preconlea phosphate buffer saline (PBS) and resusp ded in inding buffer. Then, $5 \mu \mathrm{L}$ annexin V-FITC $\mathrm{S}$ a d mixed gently, and the mix put in the $d$ fo cubation for $15 \mathrm{~min}$. In addition, $5 \mu \mathrm{L} \mathrm{PI}$ was? do the mple. The apoptotic cell rate was measui, $d w$. a flow cytometer (Beckman Coulter, USA).

\section{Migration as}

Cell migration evaluated by a modified two-chamber migratior av with ore size of $8 \mu \mathrm{m}$. A cell suspension of $100 \mu$ are $2 \times 10^{5}$ cells $/ \mathrm{mL}$ ) without serum was added to th per transwell. Then, $600 \mu \mathrm{L}$ culture medium with $10 \%$ FE was added to the lower compartment of the ell transwell. A375 cells were maintained for $24 \mathrm{~h}$ at $37^{\circ} \mathrm{C}$ th humidified air containing $5 \% \mathrm{CO}_{2}$. After incubation, $c$ s at the upper surface of the filter were removed by on swab, and the filter was fixed with methanol for 5 hin. A375 cells at the lower surface of the filter were ained by Giemsa for $15 \mathrm{~min}$. Cells were counted on a $100 \times$ microscope (Olympus CKX41, Japan).

\section{Cell transfection}

To clarify the function of $\mathrm{H} 19, \mathrm{pEX}-\mathrm{H} 19$ and its corresponding negative control (NC) pcDNA3.1 (GenePharma Co., China) were transfected into A375 cells. Pre-treated cells at the density of $2 \times 10^{5}$ cells/well were seeded and incubated until the cells arrived at $70-80 \%$ confluence, and they were then transfected with pEX-H19 or NC by Lipofectamine 2000 reagent (Invitrogen, USA).

\section{Quantitative real time polymerase chain reaction (qRT-PCR)}

Total RNA was obtained from A375 cells using Trizol reagent (Invitrogen). The One-Step SYBR ${ }^{\circledR}$ PrimeScript ${ }^{\circledR}$ PLUS RT-RNA PCR kit (TaKaRa Biotechnology, China) was used for real-time PCR analysis to determine the expression level of H19. GAPDH was the internal control for $\mathrm{H} 19$.

\section{Western blot}

Western blot was used in our study to detect protein expression. Protein was obtained from A375 cells using RIPA lysis buffer (Cat. No. R0010, Solarbio, China) supplemented with protease inhibitors (Thermo Fisher Scientific). The $\mathrm{BCA}^{\mathrm{TM}}$ protein assay kit (Pierce, USA) was used for determining proteins concentration. The western blot system was established by a Bio-Rad Bis-Tris Gel system 
following the manufacturer's instructions. Primary antibodies included: anti-cyclin D1 antibody (ab134175), antiBcl-2 antibody (ab32124), anti-Bax antibody (ab32503), anti-pro caspase 3 antibody (ab32150), anti-cleaved caspase-3 antibody (ab32042), anti-pro caspase- 9 antibody (ab32539), anti-cleaved-caspase-9 antibody (ab2324), anti-matrix metalloproteinase (MMP)-9 antibody (ab73734), anti-MMP-2 antibody (ab37150), anti-phosphatidylinositol 3'kinase (PI3K) antibody (ab191606), anti-phospho-PI3K antibody (ab182651), anti-protein kinase B (AKT) antibody (ab8805), anti-phospho-AKT antibody (ab8933), and anti- $\beta$ actin antibody (ab8227), all from Abcam (UK). Primary antibodies were prepared in $5 \%$ blocking buffer and diluted according to product instruction. These primary antibodies were incubated in membrane and maintained at $4^{\circ} \mathrm{C}$ overnight at the recommended concentration. For the second antibody incubation, cells were incubated with horseradish peroxidase (HRP) conjugated second antibody. Detection of signals and band intensity analyses were done by Image Lab $^{\mathrm{TM}}$ Software (Bio-Rad, China).

\section{Statistical analysis}

Data are reported as means $\pm S D$, based on at least three experiments. Statistical analyses were performed using Graphpad 6.0 statistical software (GraphPad, USA). $P$-values were calculated using a one-way analys of variance (ANOVA) and Student's $t$-test. $\mathrm{P}$ values $0.0^{\prime}$ were considered significant.

\section{Results}

\section{SchA decreased cell proliferation a nhanced dell apoptosis in A375 cells}

Firstly, an experiment was design to de rmine the concentration at which SchA had the rect in decreasing cell viability. As shown different concentrations of SchA $>, \nu \vee, 40$, and $50 \mu \mathrm{M}$ ) had significantly decreas abilit vith increasing concentrations of SchA comp ad y $h$ con $l(P<0.05, P<0.01$ or $\mathrm{P}<0.001)$. SchA at $\mathrm{t}$. on of $30 \mu \mathrm{M}$ was chosen for the subseque expe, onts because $50.2 \%$ cell viability was close to $E^{\prime}$ 'nder this oncentration. BrdU assay was performed to cetecl "proliferation and we found that SchA $(30 \mu \mathrm{M})$, nificantly a reased cell proliferation $(\mathrm{P}<0.01$, Figure ,). In dition, due to the close relationship between cyclin D cell cle, we found that SchA $(30 \mu \mathrm{M})$ downre d $c, 1(P<0.05$, Figure $2 \mathrm{C})$ and enhanced cell sopt is $(P<0.001$, Figure 2D). Moreover, we detected ated level of apoptotic proteins, and found tha leaved-caspase-3 $(P<0.001)$, cleaved-caspase-9 $(P<0.01)$, and $\operatorname{Bax}(P<0.01)$ were up-regulated while $\mathrm{Bcl}-2$ was down-regulated $(\mathrm{P}<0.05)$ by SchA $(30 \mu \mathrm{M})$ compared with control (Figure 2E and F). Taken together, these findings indicate that SchA decreased cell proliferation and enhanced cell apoptosis.

\section{SchA decreased cell migration in A375 cells}

In $\mathrm{MM}$, migration is one of the main approa es for tumor metastasis (14). Hence, we detected th effe of SchA $(30 \mu \mathrm{M})$ on cell migration. As shown in. $3 A$ SchA decreased cell migration $(P<0.01)$ compa control. In addition, it is well known that MMP-2 and 9 are involved in cell migration (15), a theref 2 , we determined their accumulated le In found that MMP-9 and MMP-2 ere significantly downregulated by SchA compared wi contro both $\mathrm{P}<0.05$, Figure $3 \mathrm{~B}$ and $\mathrm{C}$ ). Thus, Sc. sho rity in decreasing cell migration.

\section{SchA negatively regulate he expression of $\mathrm{H} 19$}

Previous rese has repuled that IncRNA H19 has shown tumor-p mot a effects in multiple tumors (11). In our study, we 6 r cur...ther $\mathrm{H} 19$ was dysregulated in A375 cells. Resu. showed that SchA $(30 \mu \mathrm{M})$ significantly de malatec ine expression of $\mathrm{H} 19$ in A375 cells compared vitt $(P<0.01$, Figure 4). This information hinted at H19 might participate in SchA's tumorsuppressive fects.

\section{SchA Ihibited cell proliferation and increased cell} sis through down-regulation of $\mathrm{H} 19$

$\checkmark$ identify the mechanism by which SchA regulated Il proliferation and apoptosis, pEX-H19 was transfected into A375 cells. The significant up-regulation of $\mathrm{H} 19$ after transfection with pEX-H19 indicated high transfection efficiency $(P<0.01$, Figure $5 A)$. Then, we detected the effects of overexpression of $\mathrm{H} 19$ on cell proliferation and cell apoptosis. Results showed that transfection with $\mathrm{pEX}$ $\mathrm{H} 19$ increased cell proliferation $(\mathrm{P}<0.01$, Figure $5 B)$, upregulated cyclin $D 1(P<0.05$, Figure $5 C)$, and decreased cell apoptosis $(P<0.01$, Figure $5 D)$ compared with $p E X$ group. In addition, the apoptotic proteins cleaved-caspase-3 $(P<0.05)$, cleaved-caspase-9 $(P<0.05)$, and Bax $(P<0.05)$ were down-regulated while $\mathrm{Bcl}-2$ was upregulated $(\mathrm{P}<0.05)$ after transfection with $\mathrm{pEX}-\mathrm{H} 19$ compared with the group transfected with $\mathrm{pEX}$ (Figure $5 E$ and $F$ ). In summary, we inferred that SchA decreased cell proliferation and enhanced cell apoptosis via downregulation of $\mathrm{H} 19$.

\section{SchA decreased cell migration via down-regulation of H19}

Similarly, we detected the effect of H19 overexpression on cell migration. We found that co-treatment with SchA and $\mathrm{H} 19$ overexpression increased cell migration $(P<0.05$, Figure $6 A)$ as well as MMP-2 and MMP-9 expression (both $\mathrm{P}<0.05$, Figure $6 \mathrm{~B}$ and $\mathrm{C}$ ) compared with co-treatment with SchA and transfection with $\mathrm{pEX}$. Thus, we found that SchA decreased cell migration via down-regulation of $\mathrm{H} 19$. 

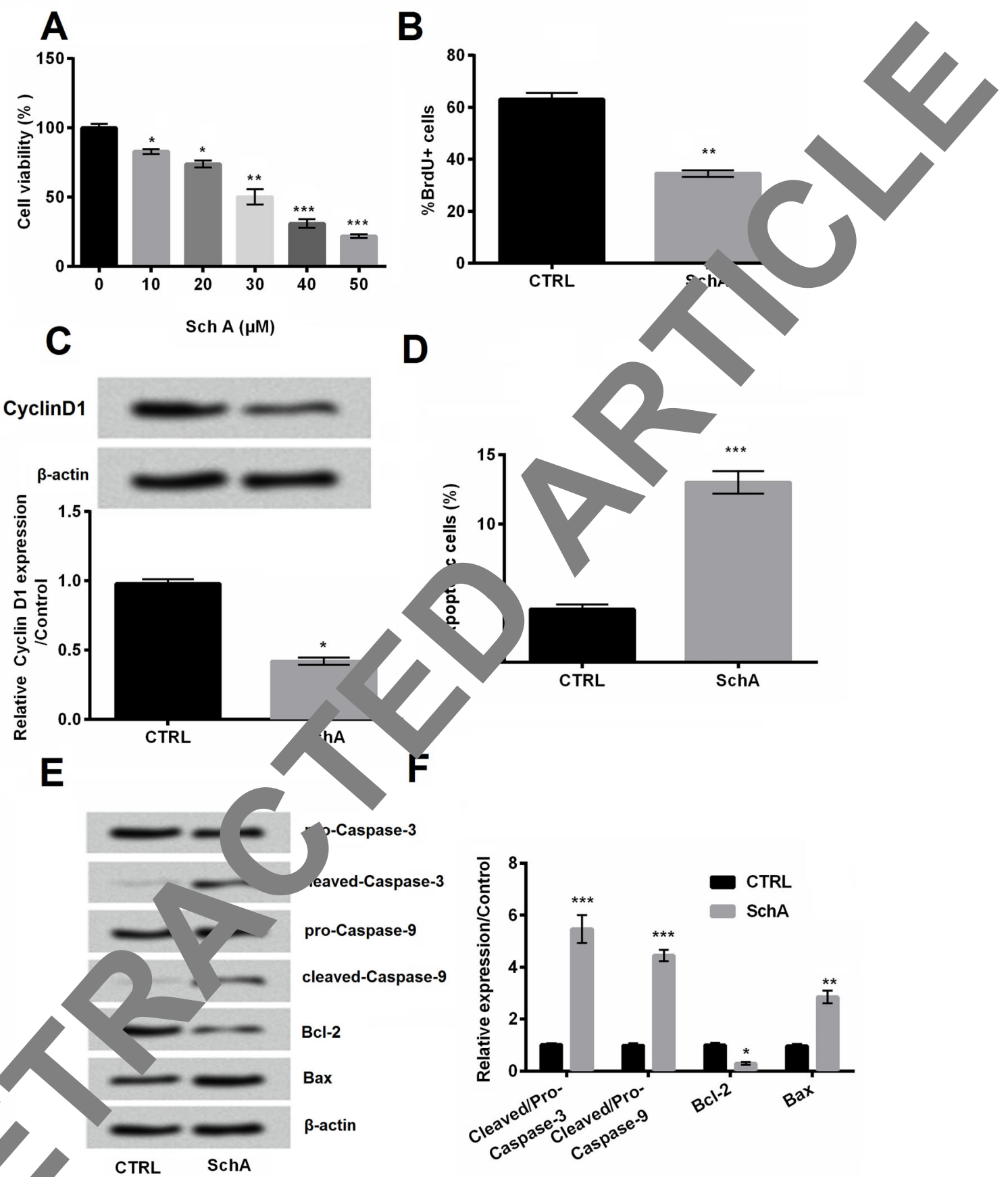

gure 2. A, Cull viability was detected under different schizandrin A (SchA) concentrations by Cell Counting Kit-8 assay. Cell olif and (C) expression and level of cyclin D1 were analyzed via BrdU, western blot, and quantitatively, respectively. D, Cell $a_{\mathrm{p}}$ osis was analyzed by flow cytometry. Cell apoptosis-related proteins were detected by western blot $(\mathbf{E})$ and quantitatively $(\mathbf{F})$. $\beta$-ack vas used as control. Data are reported as means $\pm S D(n=3)$. ${ }^{*} P<0.05$, ${ }^{* \star} P<0.01$, and ${ }^{* * *} P<0.001$ compared to control (ANOVA and Student's $t$-test). 

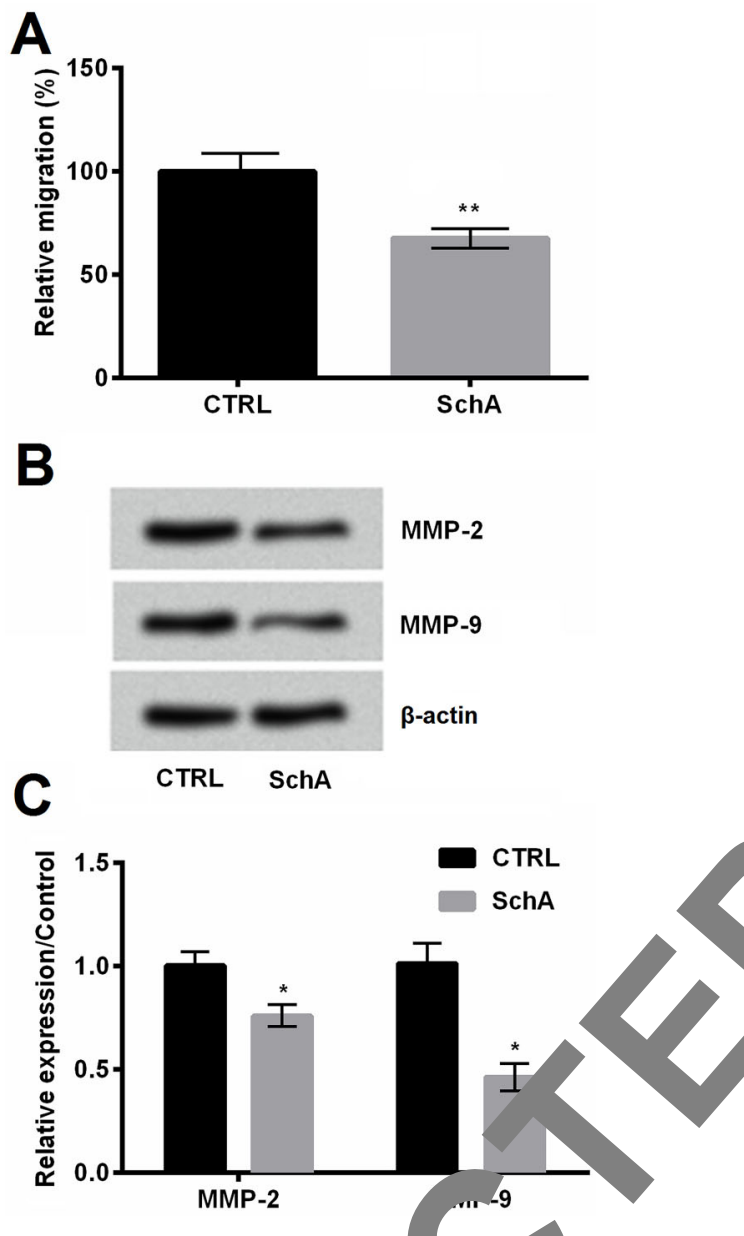

Figure 3. Schizandrin A (SchA) decrease ell migr on. A, Cell migration was detected by transwell two assay. B, MMP-2 and MMP-9 protein leve' determined by western blot. C, Levels of MMP-2 and Mi -9y nalyzed quantitatively. $\beta$-actin was used as control. are reported as means \pm SD $(n=3) .{ }^{*} P<0.05 ?{ }^{*} P<1$, compared to control (Student's $t$-test).

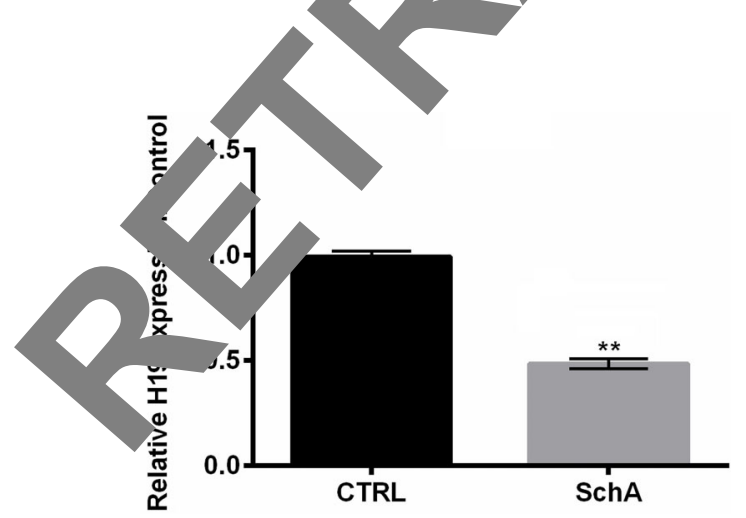

Figure 4. Schizandrin $A$ (SchA) down-regulated $\mathrm{H} 19$ in $\mathrm{A} 375$ cells via qRT-PCR. Data are reported as means $\pm S D(n=3)$. ${ }^{* \star} P<0.01$ (Student's $t$-test).

\section{SchA inactivated PI3K/AKT pathway by down-} regulation of $\mathrm{H} 19$

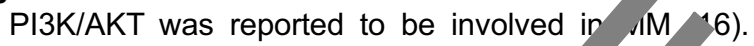
Hence, we investigated the effects of SchA o wh path way. Results demonstrated that SchA down-regu. $\mathrm{d} d$ phosphorylation of PI3K $(\mathrm{P}<0.001)$ and AKT $(\mathrm{P}<\mathrm{J5})$ while co-treatment with SchA and $\mathrm{H} 19$ o expres on led to the opposite results $(P<0.01, F \quad 7 A \quad d)$. Thus, SchA might inhibit PI3K/AKT thway through downregulation of $\mathrm{H} 19$.

\section{Discussion}

MM is a catastropuc s. cancer with aggressive biological actions, tance to hemotherapy, and poor results (2). MM as complex progress and it is hard to fully elucidate 9 mechanisms. Hence, we used A375 cells construct an in vitro cell model and investig the efte of SchA on A375 cells. Results demonst ed chA decreased cell proliferation and migration, eased cell apoptosis, and inhibited PI3K/ AKT signali pathway through down-regulating IncRNA

- line A375 is often used for the in vitro model of MM (17). I stly, we explored the effects of SchA on cell viand cell proliferation. Results showed that SchA signituantly decreased cell viability in a dose-dependent anner. Moreover, BrdU assay demonstrated that SchA significantly alleviated cell proliferation. Our study was consistent with the previous study that SchA inhibited cell proliferation in breast cancer cells (18). Furthermore, previous studies have reported that cyclin D1 is an important proto-oncogene that is upregulated in multiple cancers, such as breast, prostate, and MM $(19,20)$. Cyclin D1 exerts important functions in regulating cell proliferation via activation of cyclin-dependent kinases (19). In our study, we found that SchA inhibited the accumulation of cyclin D1, which is consistent with the findings of Kim et al. (18) that SchA decreases cell proliferation by regulating cell cycle-related proteins, such as cyclin D1.

In addition, it is well-known that apoptosis plays important roles in cancer cells, and apoptosis related proteins are considered a promising target for molecule-based pharmacological intervention (21). SchA increased cell apoptosis in our study. Moreover, caspase-3, caspase-9, $\mathrm{Bcl}-2$, and Bax are all apoptosis-related proteins (22). Our study revealed that SchA up-regulated the expression of pro-apoptotic proteins cleaved-caspase-3, cleavedcaspase-9, and Bax while it down-regulated the expression of anti-apoptotic protein Bcl-2. Our findings were consistent with the study of Kim et al. (23) that SchA extract induced apoptosis presented by the down-regulation of Bcl-2 and up-regulation of Bax, cleaved-caspase, and cleaved-caspase-9.

Furthermore, migration of cells to surrounding tissues is one of the initial and critical steps in the progression 

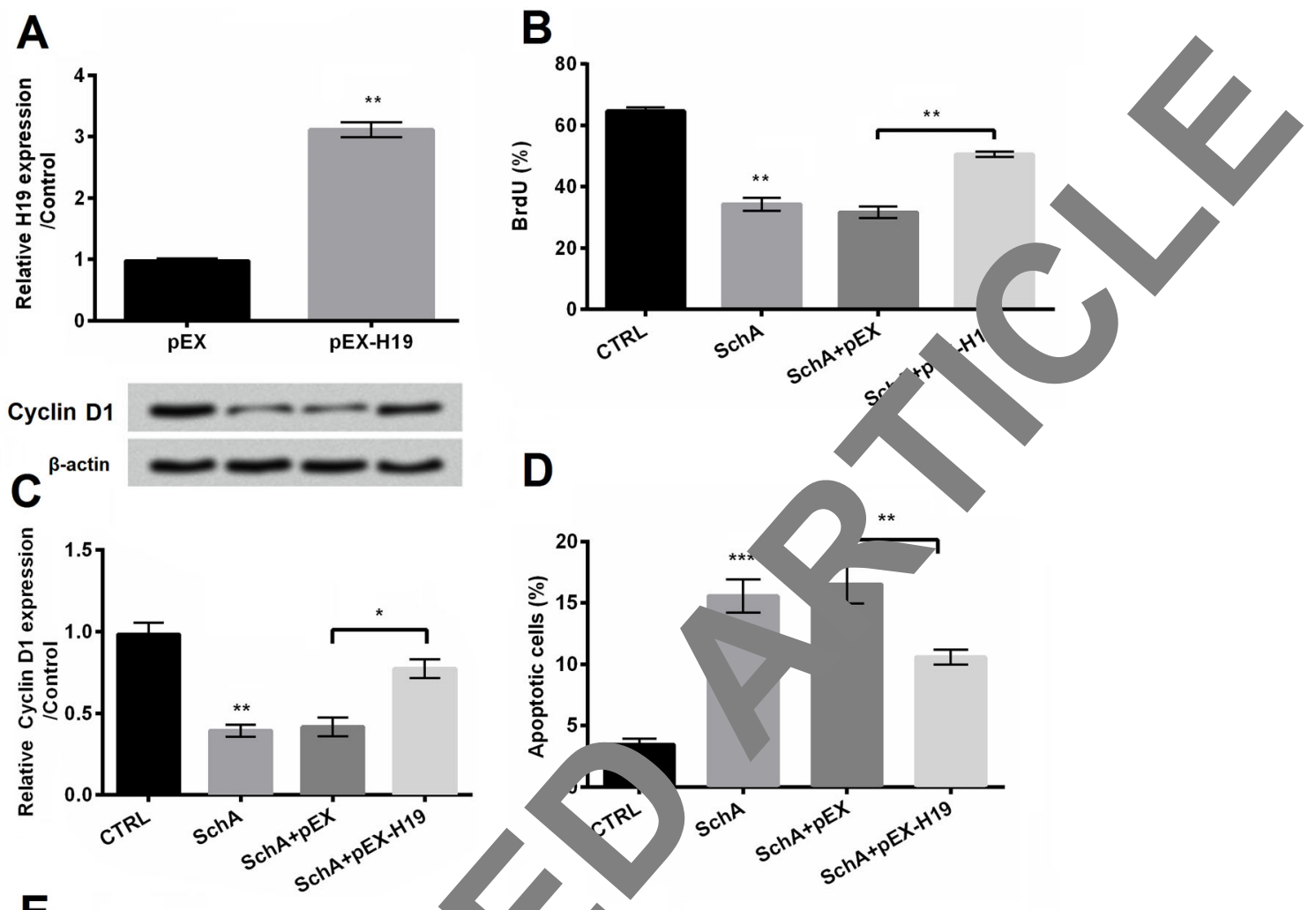

E
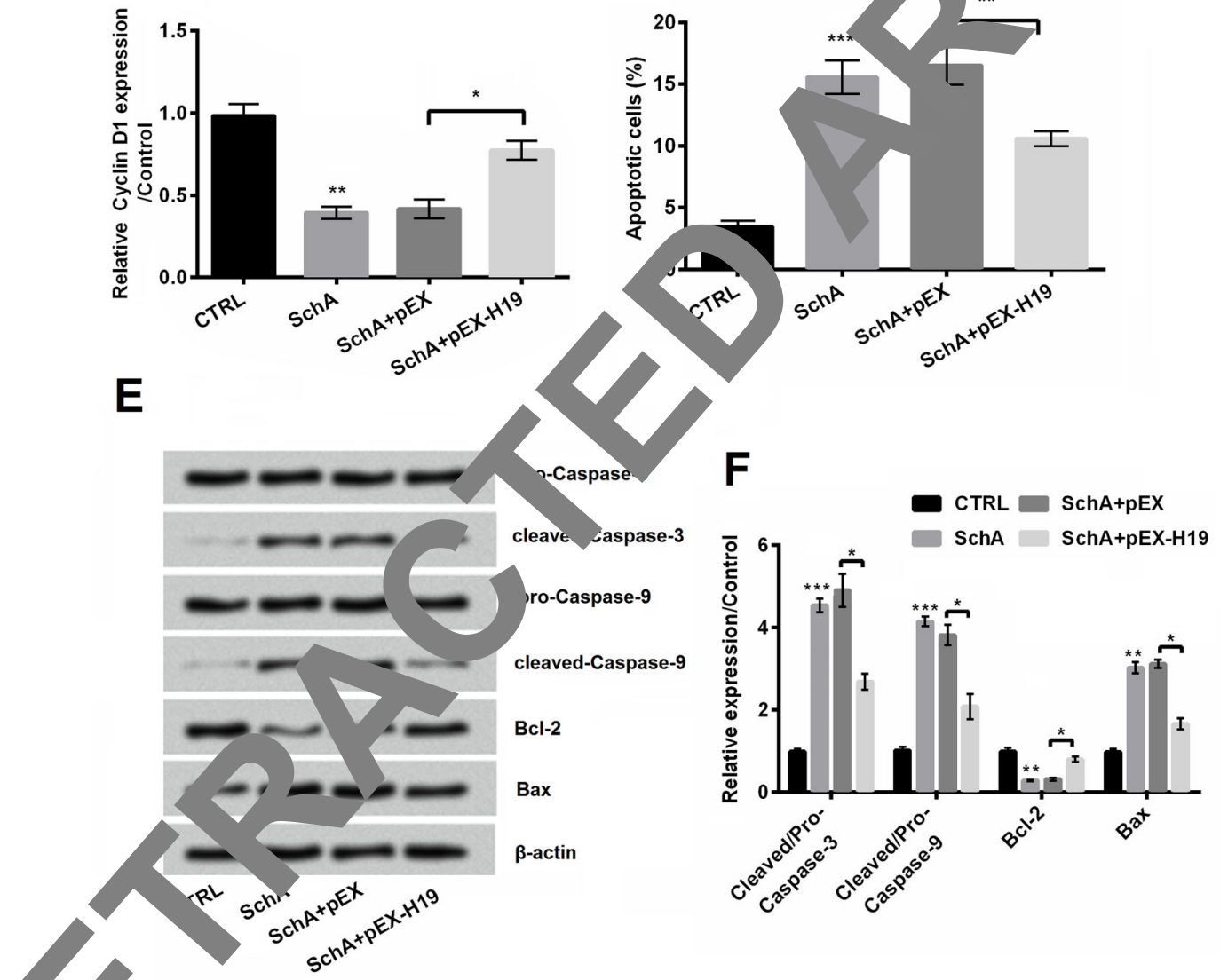

Figure 5. 'zan' A (SchA) decreased cell proliferation and increased cell apoptosis via down-regulation of H19. A, Overexpression of vas shed through transfection with pEX-H19 in A375 cells. Cell proliferation (B) and (C) expression and level of cyclin I we analyzed via BrdU, western blot, and quantitatively, respectively. D, Cell apoptosis was analyzed by flow cytometry. Cell ed proteins were detected by western blot $(\mathbf{E})$ and analyzed quantitatively $(\mathbf{F})$. $\beta$-actin was used as control. All data are re, ed as means $\pm S D(n=3)$. ${ }^{*} P<0.05,{ }^{* *} P<0.01$ and ${ }^{* \star *} \mathrm{P}<0.001$ compared to control or as indicated (ANOVA and Student's $t$-test).

of cancer metastasis. Metastasis is the most important cause of cancer-related death (24). Therefore, we detected the effects of SchA on cell migration and found that it significantly decreased cell migration, suggesting it might have functions in inhibiting MM metastasis. Moreover,
MMP-2 and MMP-9 are two important factors in cell migration and their overexpression is often related to poor progression in cancer (25). In our study, SchA decreased MMP-2 and MMP-9 expression, indicating the inhibition effects of SchA in the progress of MM. 


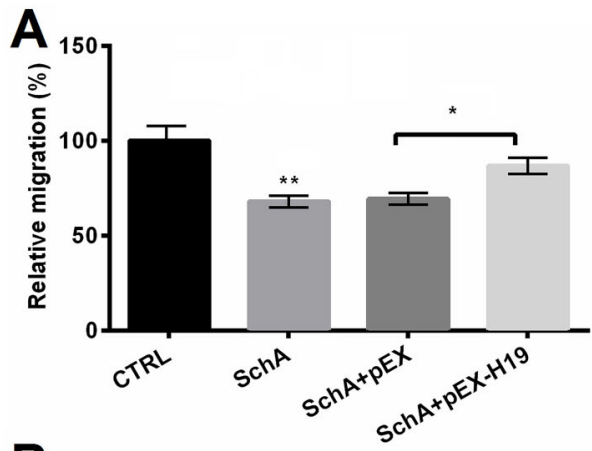

B
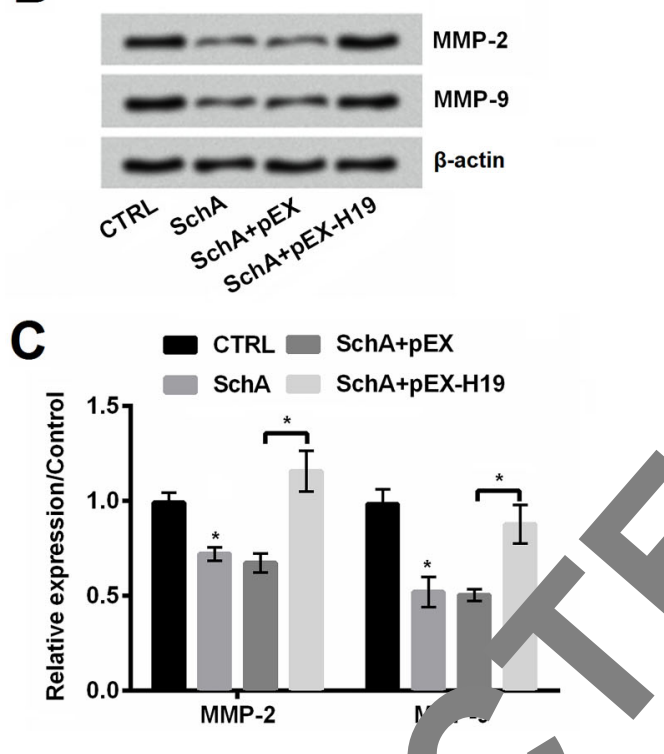

Figure 6. Schizandrin A (SchA) decrec d cell gration by down-regulating $\mathrm{H} 19$. A, Cell migration was sy transwell two-chamber assay. MMP-2 an detected by western blot (B) an anay quantitatively (C). $\beta$-actin was used as control. Data ported as means \pm SD $(n=3) .{ }^{*} P<0.05$ and ${ }^{* *} P<C \quad$ mpa to control or as indicated (ANOVA and Student's st).

Increasing e dence rggested that IncRNAs acted as molecular hes to gulate cell proliferation, cell apoptosis, and cel igration (26). Among the identified IncRNAs 119 was ow erved to be essential for human tumor swtt 27), including bladder cancer (27), colorectal canc 8), stric cancer (29), and MM (12). In our $s^{+}$ve to nat SchA inhibited the expression of $\mathrm{H} 19$, agge ina that $\mathrm{H} 19$ might be involved in the inhibition o vi hat on A375 cell growth and migration.

alter the expression of $\mathrm{H} 19, \mathrm{pEX}-\mathrm{H} 19$ was transfectea into A375 cells. The results showed that cotreatment and transfection with $\mathrm{pEX}-\mathrm{H} 19$ and SchA blockaded the effects of SchA on A375 cells as evidenced by increasing cell proliferation and migration, and inhibiting

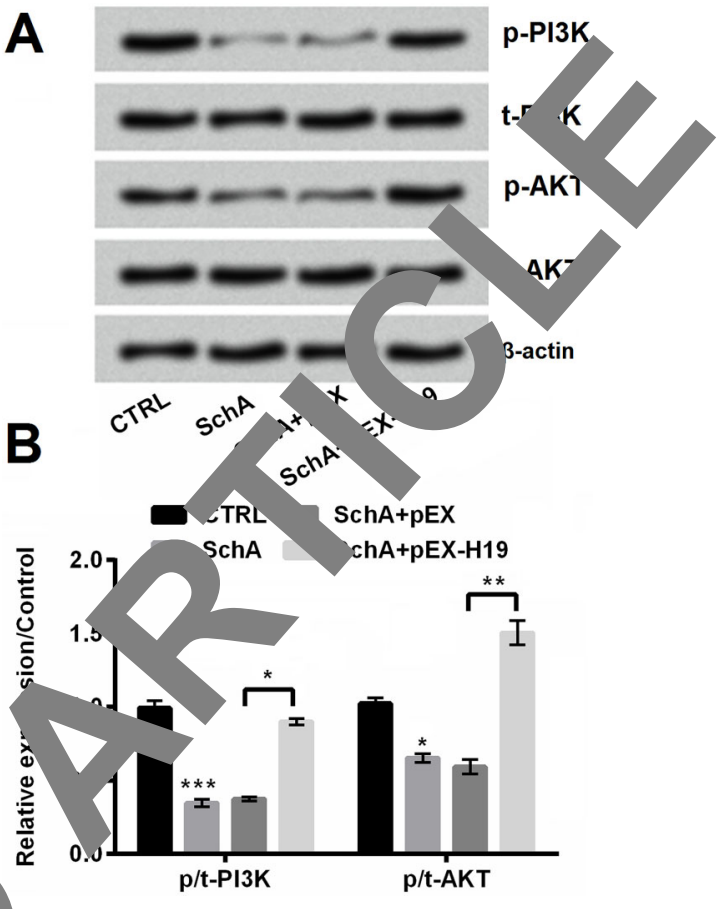

7. Schizandrin A (SchA) inhibited PI3K/AKT through egulation of H19. A, Phosphorylation of PI3K and AKT was detected by western blot. B, Levels of p/t-PI3K and p/t-AKT ere analyzed quantitatively. $\beta$-actin was used as control. Data are reported as means $\pm S D(n=3)$. ${ }^{*} P<0.05,{ }^{* *} P<0.01$, and ${ }^{* * *} \mathrm{P}<0.001$ compared to control or as indicated (ANOVA).

cell apoptosis. This is consistent with previous findings that H19 enhances breast cancer cell proliferation (30) and elevated $\mathrm{H} 19$ promotes bladder cancer proliferation (31). In addition, IncRNA H19 promotes cell migration in cholangiocarcinoma (32). Moreover, Zhu et al. (33) found that H19 knockdown increased the rate of apoptosis in ovarian cancer cells, which was the same trend as our study. Our study was the first to demonstrate that SchA played its roles in A375 cells through regulation of $\mathrm{H} 19$.

PI3K/AKT signaling pathway has been reported to play important roles in cell proliferation, survival, and metabolism (34), and was observed to be closely associated with MM (35). In our study, SchA decreased the phosphorylation level of PI3K and AKT, indicating that SchA inactivated $\mathrm{PI} 3 \mathrm{~K} / \mathrm{AKT}$ signaling pathways. On the other hand, overexpression of $\mathrm{H} 19$ activated $\mathrm{PI}$ IK/AKT pathway. Previous studies proved that activation of PI3K/AKT was often related to the promotion of cancer progression while inactivation of PI3K/AKT was involved in inhibiting cancer development (36). These results demonstrated that SchA might inhibit A375 cell growth through inhibiting PI3K/AKT pathway. 


\section{Conclusions}

Our study demonstrated that SchA inhibited proliferation and migration of melanoma A375 cells, and increased apoptosis and inhibited PI3K/AKT pathway through down-regulation of IncRNA H19. Our st $y$ may lay the foundation for the study of MM in the $f$

\section{References}

1. Bandarchi B, Jabbari CA, Vedadi A, Navab R. Molecular biology of normal melanocytes and melanoma cells. J Clin Pathol 2013; 66: 644-648, doi: 10.1136/jclinpath-2013201471.

2. Ryska A, Horky O, Berkovcova J, Ticha I, Kalinova M, Matejckova M, et al. [Malignant melanoma - from classical histology towards molecular genetic testing]. Klinicka onkologie: casopis Ceske a Slovenske Onkologicke Spolecnosti 2017; 30: 182-189, doi: 10.14735/amko2017182.

3. Atkinson V. Recent advances in malignant melanoma. Intern Med J 2017; 47: 1114-1121, doi: 10.1111/imj.13574.

4. Wang CP, Li GC, Shi YW, Zhang XC, Li JL, Wang ZW, et al. Neuroprotective effect of schizandrin A on oxygen and glucose deprivation/reperfusion-induced cell injury in primary culture of rat cortical neurons. J Physiol Biochem 2014; 70: 735-747, doi: 10.1007/s13105-014-0342-3.

5. Li S, Xie R, Jiang C, Liu M. Schizandrin A alleviates LPSinduced injury in human keratinocyte cell hacat through a microRNA-127-dependent regulation. Cell Physiol Biocher 2018; 49: 2229-2239, doi: 10.1159/000493826.

6. Kong D, Zhang D, Chu X, Wang J. Schizandrin A en chemosensitivity of colon carcinoma cells to 5-fll ure through up-regulation of miR-195. Biomed $\mathrm{Pr}$ ma 2018; 99: 176-183, doi: 10.1016/j.biopha.201 1.035.

7. Zhang ZL, Jiang QC, Wang SR. Schisar ir reverses doxorubicin-resistant human breast cancer cell lin. $v$ the inhibition of P65 and Stat3 phosphor 1. Breast o ncer 2018; 25: 233-242, doi: 10.1007/s1 82-017-0?21-9.

8. Shi X, Sun M, Liu H, Yao Y, Song Y. I non-c ling RNAs: a new frontier in the study of human 2013; 339: 159-166, doi: 10 cli canlet.zu13.06.013.

9. Schmitt AM, Chang HY. Lon nory RNAs in cancer pathways. Cancer Cell 2016; $+52-463$, doi: 10.1016/ j.ccell.2016.03.010.

10. Yang J, Li C, Mud A, G X. Lnc .NA PVT1 predicts prognosis and regulate in 1 in prostate cancer. Biosci Biotechnol B hem 17; 81: 2301-2306, doi: 10.1080/ 09168451 138704 。

11. Matouk N, Rc a, Abu-lail R, Mezan S, Gilon M, Gerst $\mathrm{E}$, et a. Oncofetal H19 RNA promotes tumor me' asir Biochim Biophys Acta 2014; 1843: 1414-1426, do " 0 ' 6/.j.b" amcr.2014.03.023.

12. Luar Zho', $\mathrm{Ni}$ X, Xia Y, Wang J, Yan Y, et al. Long noning $\mathrm{H} 19$ promotes glucose metabolism and cell with in malignant melanoma via miR-106a-5p/E2F3 axis. Camur Res Clin Oncol 2018; 144: 531-542, doi: 10.1007/ ר0432-018-2582-z.

13. S. G, Li H, Gao F, Tan Q. IncRNA H19 predicts poor prognosis in patients with melanoma and regulates cell growth, invasion, migration and epithelial-mesenchymal transition in melanoma cells. Onco Targets Ther 2018; 11: 3583-3595, doi: 10.2147/OTT.S160143.
14. van Roosmalen W, Le Devede Golâ. Smid M, Pulyakhina I, Timmermans AM al. Tumor cell migration screen identifies SRPK1 as east $c$ cer metastasis determinant. J Clin Inves 15, 10. $1172 / \mathrm{JCl} 74440$.

15. Piromkraipak $P$, San airoj $K$, 'otai $W$, Chaithirayanon $\mathrm{K}$, Unchern S, S av P, et ar. Cysteinyl leukotriene receptor antagonists inhibı rration, invasion, and expression of MMP_ uman glioslastoma. Moll Cell Neurobiol 2018; 38: 5 ,-57' doi-10.1007/s10571-017-0507-z.

16. Meng $F,{ }, X$, Wang J, Wang Z. Clinical significance on $R-138$ in patients with malignant melanon ah targ ing of PDK1 in the PI3K/AKT autophagy signá $9 \mathrm{p}$. Oncol Rep 2017; 38: 1655-1662, doi: 10. 3892/c 1.5838 .

17. Liu XY, ng H, Yang ZG, Wang XY, Ruan LM, Fang DR, et al. Murine inhibits invasiveness and metastasis of man malignant melanoma cell line A375 in vitro. Int $J$ matol 2008; 47: 448-456, doi: 10.1111/j.1365-4632. 8.03627.x.

IIm SJ, Min HY, Lee EJ, Kim YS, Bae K, Kang SS, et al. Growth inhibition and cell cycle arrest in the G0/G1 by schizandrin, a dibenzocyclooctadiene lignan isolated from Schisandra chinensis, on T47D human breast cancer cells. Phytother Res 2010; 24: 193-197, doi: 10.1002/ptr.2907.

19. Mukhopadhyay A, Banerjee S, Stafford LJ, Xia C, Liu M, Aggarwal BB. Curcumin-induced suppression of cell proliferation correlates with down-regulation of cyclin D1 expression and CDK4-mediated retinoblastoma protein phosphorylation. Oncogene 2002; 21: 8852-8861, doi: 10.1038/ sj.onc.1206048.

20. Florenes VA, Faye RS, Maelandsmo GM, Nesland JM, Holm R. Levels of cyclin D1 and D3 in malignant melanoma: deregulated cyclin D3 expression is associated with poor clinical outcome in superficial melanoma. Clin Cancer Res 2000; 6: 3614-3620.

21. Hassan $M$, Watari $H$, AbuAlmaaty A, Ohba $Y$, Sakuragi $N$. Apoptosis and molecular targeting therapy in cancer. BioMed Res Int 2014; 2014: 150845, doi: 10.1155/2014/ 150845.

22. Liang S, Sun K, Wang Y, Dong S, Wang C, Liu L, et al. Role of Cyt-C/caspases-9,3, Bax/Bcl-2 and the FAS death receptor pathway in apoptosis induced by zinc oxide nanoparticles in human aortic endothelial cells and the protective effect by alpha-lipoic acid. Chem Biol Interact 2016; 258: 40-51, doi: 10.1016/j.cbi.2016.08.013.

23. Kim HS, Lee JH, Park HS, Lee GS, Kim HW, Ha KT, et al. Schizandra chinensis extracts induce apoptosis in human gastric cancer cells via JNK/p38 MAPK activation and the ROS-mediated/mitochondria-dependent pathway. Pharm Biol 2015; 53: 212-219, doi: 10.3109/13880209.2014.91 3297. 
24. Duff $D$, Long A. Roles for RACK1 in cancer cell migration and invasion. Cell Signal 2017; 35: 250-255, doi: 10.1016/ j.cellsig.2017.03.005.

25. Roomi MW, Monterrey JC, Kalinovsky T, Rath M, Niedzwiecki A. Patterns of MMP-2 and MMP-9 expression in human cancer cell lines. Oncol Rep 2009; 21: 1323-1333, doi: 10.3892/or_00000358.

26. Kumar MM, Goyal R. LncRNA as a therapeutic target for angiogenesis. Curr Top Med Chem 2017; 17: 1750-1757, doi: $10.2174 / 1568026617666161116144744$.

27. Luo M, Li Z, Wang W, Zeng Y, Liu Z, Qiu J. Long non-coding RNA H19 increases bladder cancer metastasis by associating with $\mathrm{EZH} 2$ and inhibiting E-cadherin expression. Cancer Lett 2013; 333: 213-221, doi: 10.1016/j.canlet.2013.01.033.

28. Tsang WP, Ng EK, Ng SS, Jin H, Yu J, Sung JJ, et al. Oncofetal H19-derived miR-675 regulates tumor suppressor RB in human colorectal cancer. Carcinogenesis 2010; 31: 350-358, doi: 10.1093/carcin/bgp181.

29. Yang F, Bi J, Xue X, Zheng L, Zhi K, Hua J, et al. Upregulated long non-coding RNA H19 contributes to proliferation of gastric cancer cells. FEBS J 2012; 279: 3159-3165, doi: 10.1111/j.1742-4658.2012.08694.x.

30. Berteaux N, Lottin S, Monte D, Pinte S, Quatannens B, Coll J, et al. H19 mRNA-like noncoding RNA promotes breast cancer cell proliferation through positive control by E2F1. J Biol Chem 2005; 280: 29625-29636, doi: 10.1074/jbc.M504033200.
31. Luo M, Li Z, Wang W, Zeng Y, Liu Z, Qiu J. Upregulated H19 contributes to bladder cancer cell proliferation by $r$ julating ID2 expression. FEBS J 2013; 280: 1709-17, do 10. 1111/febs.12185.

32. Wang WT, Ye H, Wei PP, Han BW, He B, Che, et LncRNAs H19 and HULC, activated by oxidative s, promote cell migration and invasion ir olangiocarciroma through a ceRNA manner. $J$ Hematol o, 201 9: 117, doi: 10.1186/s13045-016-0348-0

33. Zhu Z, Song L, He J, Sun Y, Liu Zou X. F-topic expressed long non-coding RNA H19 cc ributes malignant cell behavior of ovarian cano Int Vlin xp Pathol 2015; 8: 10082-10091.

34. Yu JS, Cui W. Prol tion, sur and metabolism: the role of PI3K/AKT/ O, analling in pluripotency and cell fate determination. Devel ent 2016; 143: 3050-3060, doi: $10.1242 /$ v. 075 .

35. Davies MA ne ro af the PI3K-AKT pathway in melanoma. Cancer J 2 o. 14_ 2147 , doi: 10.1097/PPO.0b013e3 $1824 d 448 c$.

36. Liu M, Ban, K, Smalley KS, Brafford P, Qiu R, et al. Notcl sig promotes primary melanoma progression by act g mitogen-activated protein kinase/phosphatidylinositol kinase-Akt pathways and up-regulating N-cadherin exp ession. Cancer Res 2006; 66: 4182-4190, doi: 